\title{
RELATIONS BETWEEN KINDRED RIEMANNIAN $P$ AND $Q$ FUNCTIONS *
}

BY D. R. CURTISS

1. Definitions and Properties of Riemannian Functions. A Riemannian $P$ function, as the term is used by Klein $\dagger$ and others, is a generalized hypergeometric function, i.e., a solution of a homogeneous linear differential equation of second order having but three singular points, all of which are regular. A $Q$ function is a hypergeometric function which has also apparently singular points, that is, points for which the function is not singular, but which are singular points of the linear differential equation of second order satisfied by the $Q$ function. A linear transformation carries the three regular singular points into the points $0, \infty, 1$, and the differential equation of a $Q$ function has then the form, as given in my thesis, $\ddagger$

$$
\frac{d^{2} Q}{d x^{2}}+p \frac{d Q}{d x}+q Q=0
$$

where

$$
\begin{aligned}
& p=\left[\frac{1-\lambda^{\prime}-\lambda^{\prime \prime}}{x}+\frac{1-\nu^{\prime}-\nu^{\prime \prime}}{x-1}+\sum_{i=1}^{i=k} \frac{1-\sigma_{i}{ }^{\prime}-\sigma_{i}{ }^{\prime \prime}}{x-s_{i}}\right], \\
& q=\frac{1}{x(x-1)}\left[-\frac{\lambda^{\prime} \lambda^{\prime \prime}}{x}+\mu^{\prime} \mu^{\prime \prime}+\frac{\nu^{\prime} \nu^{\prime \prime}}{x-1}+\sum_{i=1}^{i=k} \frac{A_{i}}{x-s_{i}}\right] .
\end{aligned}
$$

The sum of the constants $\lambda, \mu, \nu$, must be 1,0 , or a negative integer, and, as a consequence of the condition that the points $s_{i}$ are not to be singular points of solutions, it follows that the $\sigma$ 's are zero or positive integers and the accessory parameters $A_{i}$ verify a system of $k$ equations each of which is quadratic or of higher degree in the $A_{i}$.

The symbol

$$
Q\left(\begin{array}{l}
\lambda^{\prime}, \mu^{\prime}, \nu^{\prime}, \\
\lambda^{\prime \prime}, \mu^{\prime \prime}, \nu^{\prime \prime}, x
\end{array}\right)
$$

* Presented to the Society, April 14, 1922.

$\dagger$ See especially Klein's lithographed lectures, Ueber die Hypergeometrische Function. Both the terms $P$ function and $Q$ function were used by Riemann, Werke, p. 67 and p. 323.

$\ddagger$ Binary families in a triply connected region, Memoirs of the AMERICAN ACADEMY, vol. 13 (1904), No. 1. 
is used to denote any $Q$ function which is a solution of (1). The numbers $\lambda^{\prime}$ and $\lambda^{\prime \prime}$ are called the exponents of the singular point $x=0$, for reasons familiar in the theory of linear differential equations; $\mu^{\prime}$ and $\mu^{\prime \prime}$ are the exponents of $x=\infty$; and $\nu^{\prime}$ and $\nu^{\prime \prime}$ are the exponents of $x=1$. If the sum of these exponents is $1-n$, the function is said to be of order $n$.

For a $P$ function the terms in $\left(x-s_{i}\right)$ disappear from the differential equation, and the sum of the exponents is 1 . A $P$ function is, then, a $Q$ function of order zero, and the family of $P$ functions satisfying a differential equation (1) free of terms in $\left(x-s_{i}\right)$ is completely determined by its exponents.

In Gauss's celebrated memoir on special hypergeometric functions, ${ }^{*}$ it is shown that between every three such functions that are kindred, that is, whose corresponding exponents differ by integers or zero, there exists a relation linear and homogeneous in the functions, with coefficients that are polynomials in $x$. Riemann showed the fundamental importance of the monodromic group in these questions. Gauss's kindred functions are functions that have the same monodromic group. By the use of this conception Riemann gave a new proof of the theorem of Gauss for $P$ functions, and was able to assign an upper limit for the degree of each coefficient.

In my thesis $\dagger I$ have extended this theorem to the case of three $Q$ functions

$$
Q_{i}\left(\begin{array}{c}
\lambda_{i}{ }^{\prime}, \mu_{i}{ }^{\prime}, \nu_{i}{ }^{\prime}, \\
\lambda_{i}{ }^{\prime \prime}, \mu_{i}{ }^{\prime \prime}, \nu_{i}^{\prime \prime}
\end{array}, x\right), \quad(i=1,2,3),
$$

which are kindred in the sense that they have the same monodromic group. In order that this be the case, corresponding exponents must be equal or differ by integers, but this condition alone is not sufficient. If, however, the $Q$ functions belong to irreducible families, i.e., families of which no member is a solution of a homogeneous linear differential equation of the first order with single-valued coefficients, they will have the same group if corresponding exponents are equal or differ by integers.

* Disquisitiones generales circa seriem infinitam, Werke, vol. 3, p. 123 . $\dagger$ Loc. cit., p. 48. 
Let us write

$$
\begin{aligned}
& \Delta_{1} \lambda=\left|\left(\lambda_{2}{ }^{\prime}-\lambda_{2}{ }^{\prime \prime}\right)-\left(\lambda_{3}{ }^{\prime}-\lambda_{3}{ }^{\prime \prime}\right)\right|, \\
& \Delta_{2} \lambda=\left|\left(\lambda_{3}{ }^{\prime}-\lambda_{3}{ }^{\prime \prime}\right)-\left(\lambda_{1}{ }^{\prime}-\lambda_{1}{ }^{\prime \prime}\right)\right|, \\
& \Delta_{3} \lambda=\left|\left(\lambda_{1}{ }^{\prime}-\lambda_{1}{ }^{\prime \prime}\right)-\left(\lambda_{2}{ }^{\prime}-\lambda_{2}{ }^{\prime \prime}\right)\right|,
\end{aligned}
$$

and similarly for $\Delta_{1} \mu, \Delta_{2} \mu, \Delta_{3} \mu, \Delta_{1} \nu, \Delta_{2} \nu, \Delta_{3} \nu$. Then if the three families are irreducible and are of order $n_{1}, n_{2}, n_{3}$, respectively, three kindred functions (2) satisfy an identity

$$
\phi_{1} Q_{1}+\phi_{2} Q_{2}+\phi_{3} Q_{3} \equiv 0,
$$

where $\phi_{1}, \phi_{2}, \phi_{3}$ are polynomials of degrees not greater than

$$
\begin{aligned}
& \frac{1}{2}\left(\Delta_{1} \lambda+\Delta_{1} \mu+\Delta_{1} \nu+n_{2}+n_{3}-2\right), \\
& \frac{1}{2}\left(\Delta_{2} \lambda+\Delta_{2} \mu+\Delta_{2} \nu+n_{3}+n_{1}-2\right), \\
& \frac{1}{2}\left(\Delta_{3} \lambda+\Delta_{3} \mu+\Delta_{3} \nu+n_{1}+n_{2}-2\right),
\end{aligned}
$$

respectively. To each function $Q_{\mathrm{i}}$ of the first family there correspond functions $Q_{2}$ and $Q_{3}$ of the other families which verify (3). From the way in which (3) is derived it follows that $\phi_{1}$ cannot vanish identically unless there is a singlevalued function $F_{1}$ such that for every function $Q_{2}$ of the second family the corresponding $Q_{3}$ of the third verifies $Q_{3}=F_{1} Q_{2}$. Similarly as regards the identical vanishing of $\phi_{2}$ or $\phi_{3}$.

In particular, we may take $Q_{2}$ and $Q_{3}$ as $P$ functions, and it follows that every irreducible $Q$ function can be expressed as a linear function with rational coefficients of two $P$ functions. Or again, we have a similar expression for a $Q$ function in terms of a $P$ function and its derivative. The formulas (4) for the degrees of the coefficients $\phi$ in (3) enable us to supply for two well known theorems regarding relations between kindred $P$ and $Q$ functions proofs that have hitherto been lacking.

2. A Linear Expression for a $Q$ Function in Terms of a $P$ Function and its Derivative, with Polynomial Coefficients. In Riemann-Weber's Die Partiellen Differentialgleichungen der Mathematischen Physik, ${ }^{*}$ there is given a brief account of some of the properties of $P$ and $Q$ functions. Thus we find on page 44

* Vol. 2, pp. 40-54. 


$$
\begin{aligned}
x^{\delta}(1-x)^{\epsilon} Q\left(\begin{array}{c}
\alpha, \beta, \gamma, \\
\alpha^{\prime}, \beta^{\prime}, \gamma^{\prime},
\end{array}\right) \\
\quad=\bar{Q}\left(\begin{array}{c}
\alpha+\delta, \beta-\delta-\epsilon, \gamma+\epsilon, \\
\alpha^{\prime}+\delta, \beta^{\prime}-\delta-\epsilon, \gamma^{\prime}+\epsilon,
\end{array}, x\right),
\end{aligned}
$$

where $\delta$ and $\epsilon$ are any numbers we please. The function $\bar{Q}$ has the same apparently singular points with the same exponents as $Q$, but not, in general, the same accessory parameters. Also (p. 51), if

we have

$$
P=P\left(\begin{array}{c}
\alpha, \beta, \gamma, x \\
\alpha^{\prime}, \beta^{\prime}, \gamma^{\prime},
\end{array}\right)
$$

$$
\frac{d P}{d x}=Q_{1}\left(\begin{array}{c}
\alpha-1, \beta+1, \gamma-1, \\
\alpha^{\prime}-1, \beta^{\prime}+1, \gamma^{\prime}-1,
\end{array}\right),
$$

but here we need the qualification (not mentioned in RiemannWeber) that none of the exponents for $P$ is zero. Combining (5) with (7), we obtain

$$
x(1-x) \frac{d P}{d x}=\bar{Q}\left(\begin{array}{c}
\alpha, \beta-1, \gamma, x \\
\alpha^{\prime}, \beta^{\prime}-1, \gamma^{\prime},
\end{array}\right) .
$$

It is then remarked that if $A(x)$ and $B(x)$ are polynomials of degrees $n-1$ and $n$ respectively, with no zeros at 0 or 1 , the sum

$$
A(x) x(1-x) \frac{d P}{d x}+B(x) P
$$

is in general a $Q$ function of order $2 n$ with the exponent scheme

$$
Q\left(\begin{array}{c}
\alpha, \beta-n, \gamma, x \\
\alpha^{\prime}, \beta^{\prime}-n, \gamma^{\prime},
\end{array}\right) .
$$

We may, however, choose $A(x)$ and $B(x)$ so that (9) is a $Q$ function of order $2 n-1$ whose symbol is

$$
Q\left(\begin{array}{c}
\alpha+1, \beta-n, \gamma, x \\
\alpha^{\prime}, \quad \beta^{\prime}-n, \gamma^{\prime},
\end{array}\right) .
$$

The question is then raised as to whether every $Q$ function whose symbol is (10) or (11) can be expressed in form (9), and a proof is suggested by a count of constants in (9) and in the general $Q$ function (10) or (11). This is unconvincing, however, because the $2 n$ effective constants in (9) correspond to $2 n$ possible apparently singular points in (10), for example, 
and the question as to whether the accessory parameters $A$ can be determined is unanswered.

Let us now attack this problem with the aid of (3) and (4), under the restriction that the families concerned are irreducible. In (3), let

$$
Q_{1}=Q, \quad Q_{2}=-\bar{Q}, \quad Q_{3}=-P,
$$

as in (10), (8) and (6). Then all the numbers $\Delta \lambda, \Delta \mu, \Delta \nu$ are zero, and the degrees of $\phi_{1}, \phi_{2}$ and $\phi_{3}$ are less than or equal to $0, n-1$ and $n$, respectively. Thus we have

$$
C Q=\phi_{2}(x) x(1-x) \frac{d P}{d x}+\phi_{3}(x) P,
$$

where the degrees of $\phi_{2}$ and $\phi_{3}$ are not greater than $n-1$ and $n$ respectively. The constant $C$ cannot vanish, since a relation

$$
P=F_{1} x(1-x) \frac{d P}{d x},
$$

where $F_{1}$ is a single-valued function of $x$, cannot be verified by all the members of a $P$ family. Division of (12) by $C$ gives the desired representation for the $Q$ function (10) by the expression (9). The same result follows if the $Q$ function (11) is used instead of (10).

This representation holds even when one or more of the exponents in (6) is zero. To prove this we consider the families

$$
\begin{aligned}
& x^{\delta}(1-x)^{\epsilon} P, \\
& x^{\delta}(1-x)^{\epsilon} Q,
\end{aligned}
$$

where $P$ has the symbol (6) and $Q$ the symbol (10) or (11), the numbers $\delta$ and $\epsilon$ being so chosen that in the new families no exponent is zero. For these functions (12) becomes, after division by $C$,

$$
\begin{array}{r}
x^{\delta}(1-x)^{\epsilon} Q=\phi_{2}(x) x(1-x) \frac{d}{d x}\left[x^{\delta}(1-x)^{\epsilon} P\right] \\
+\phi_{3}(x) x^{\delta}(1-x)^{\epsilon} P,
\end{array}
$$

where $\phi_{2}$ and $\phi_{3}$ are polynomials in $x$ of degrees not greater than $n-1$ and $n$ respectively. If we carry out the indicated differentiation, rearrange terms and divide by $x^{\delta}(1-x)^{\epsilon}$, we have 


$$
Q=\phi_{2}(x) x(1-x) \frac{d P}{d x}+\left\{\phi_{2}(x)[\delta(1-x)-\epsilon x]+\phi_{3}(x)\right\} P,
$$

which is of the form desired.

There remains the question whether we can dispense with the assumption that the families are irreducible. In my thesis I have shown that there are reducible $Q$ families which do not have the same group as any $P$ family. Obviously no such $Q$ family (10) or (11) can be expressed in form (9), but particular members might be so expressed in terms of particular $P$ functions. However, I have shown in my thesis that formula (3) holds for any three families having the same group, and (4) holds also when there are no semisingular points. Hence the representation we are discussing holds even in reducible cases provided $Q$ and $P$ have the same group and have no semisingular points.

3. A Linear Expression for a $Q$ Function in Terms of $P$ Functions, with Constant Coefficients. The other theorem, for which I have found no previous proof other than a count of constants, equally unconvincing, is that of Klein * which states that to form a $Q$ function of order $\kappa$ in the most general way we must make a linear combination, with constant coefficients, of $\kappa+1$ kindred $P$ functions. The statement is somewhat ambiguous; however, we shall now show that it is true, in the sense that every $Q$ function is so expressible, with reservations in certain reducible cases as in $\S 2$. This answers a question raised on page 49 of my thesis.

We will first suppose the $Q$ family irreducible. If of even order we use the notation (10), if of odd order, (11), and express $Q$, by formulas (3) and (4), in terms of the two kindred $P$ families, $P$ and $\bar{P}$, where $P$ has the symbol (6), and $\bar{P}$ the symbol

This gives us

$$
\bar{P}\left(\begin{array}{l}
\alpha+1, \beta, \gamma, x \\
\alpha^{\prime}-1, \beta^{\prime}, \gamma^{\prime}
\end{array}\right)
$$

$$
C Q=\phi_{2}(x) \bar{P}+\phi_{3}(x) P,
$$

where $C$ is a constant, and $\phi_{2}$ and $\phi_{3}$ are polynomials in $x$ of

* Hypergeometrische Funktionen, p. 233. 
degrees not greater than $n-1$ and $n$ respectively in case $Q$ $i_{S}$ of form (10), and of degrees not greater than $n-1$ each if $Q$ is of form (11). The form of the exponents at zero shows that $\bar{P}$ cannot be equal to $P$ multiplied by a single valued function $F$, hence $C$ is not zero and we can divide through by C. A typical term of the product $\phi_{3}(x) P$ is

$$
a_{k} x^{k} P\left(\begin{array}{l}
\alpha, \beta, \gamma, x \\
\alpha^{\prime}, \beta^{\prime}, \gamma^{\prime}, x
\end{array}\right)=a_{k} P_{k}\left(\begin{array}{l}
\alpha+k, \beta-k, \gamma, x \\
\alpha^{\prime}+k, \beta^{\prime}-k, \gamma^{\prime}, x
\end{array}\right),
$$

where $a_{k}$ is a constant; similarly the product $\phi_{2}(x) \bar{P}$ is a sum of terms $b_{k} \vec{P}_{k}$. Thus $Q$ is expressed in the desired form

if $Q$ is of order $2 n$, or

$$
Q=\sum_{k=0}^{n-1} b_{k} \bar{P}_{k}+\sum_{k=0}^{n} a_{k} P_{k}
$$

$$
Q=\sum_{k=0}^{n-1} b_{k} \bar{P}_{k}+\sum_{k=0}^{n-1} a_{k} P_{k}
$$

if $Q$ is of order $2 n-1$, where the $a$ 's and $b$ 's are constants and the functions $\bar{P}_{k}$ and $P_{k}$ are $P$ functions as indicated in (15).

As in $\S 2$, we need additional hypotheses in case $Q$ is reducible. Our proof is then not valid unless the families $Q, P, \bar{P}$ have the same group (which does not follow merely because corresponding exponents differ by integers) and no point is sem singular. In my thesis (p. 59) I have considered the exceptional cases for a $Q$ function of order 1 , and have shown that such a $Q$ function can always be expressed linearly in terms of two kindred $P$ functions if its monodromic group is possible for $P$ functions. This proof requires the examination of various types and classes of reducible groups, and we shall not attempt here to discuss the corresponding problem for $Q$ functions of order higher than 1.

As a consequence of Klein's theorem it follows that every $\kappa+2$ irreducible $Q$ functions of order $\kappa$ having the same regular singular points and the same exponents at these points, but whose accessory parameters may differ, are linearly dependent; and this conclusion holds in certain reducible cases.

These theorems and methods of proof admit generalizations to the solutions of homogeneous linear differential equations of order $n$.

NORTHWESTERN UNIVERSITY 